\title{
Nucleon Resonance Excitation with CLAS
}

\author{
Raffaella De Vita \\ (for the CLAS Collaboration) \\ Istituto Nazionale di Fisica Nucleare, Sezione di Genova \\ Via Dodecaneso 33, 16146 Genova, Italy
}

Received on 3 November, 2003

\begin{abstract}
The study of the baryon spectrum is a fundamental part of the scientific program in Hall B at Jefferson Laboratory. The so called $\mathrm{N}^{*}$ program indeed concerns the measurement of the electromagnetic production of exclusive hadronic final states, with the purpose of extracting information on baryon excited states. CLAS, the CEBAF Large Acceptance Spectrometer, is explicitly designed for conducting a broad experimental program in hadronic physics, using the continuous electron beam provided by the laboratory. An overview of the most recent results is presented.
\end{abstract}

\section{Introduction}

The study of the nucleon structure is one of the primary interests in strong interaction physics and has been the subject of experimental and theoretical studies for several decades. One of the primary manifestations of the complex internal structure of the nucleon is the existence of its excited states, i.e. baryon resonances. These play an important role in intermediate energy phenomena and understanding their nature is a necessary step to reach a comprehensive picture of strong interaction physics.

The excited states of the nucleon were first observed in $\pi N$ scattering in which their contribution was clearly evident as bumps in the total cross section. These measurements allowed a first classification of the excitation spectrum of the nucleon, providing measurement of the masses, widths, quantum numbers, and branching ratios of many baryon resonances [1]. In spite of the large amount of information collected by these experiments, the number of states that were identified was less than what predicted by the standard quark model. A possible explanation is that such "missing" states may decouple from the $\pi N$ channel, making them undetectable in experiments with pion beams. Other explanations come from theoretical models that are able to predict a smaller number of states based on a reduced set of degrees of freedom [2]. Unraveling this problem requires measurements with probes different from pion beams.

The construction of new high intensity and high duty cycle electron and photon facilities opened new possibilities for the study of baryon resonances using electromagnetic probes. These provide information on the resonance and nucleon wavefunctions through the measurement of the helicity amplitudes, i.e. the photocouplings for $\gamma N \rightarrow N^{*}$ vertices. Furthermore electroproduction allows us to explore baryon structure for different distance scales by varying the photon virtuality.

Nowadays electroexcitation processes are a fundamental tool to pursue these studies. However due to the complex- ity of the baryon spectrum, the proximity and overlapping nature of the various excited states, the measurement of a single channel is not sufficient to complete this research program. On the contrary a thorough study of resonance properties requires the measurement of cross sections, angular distributions, as well as polarization observables for different final states.

A broad experimental program for the study of baryon resonances is in progress in Hall B of Jefferson Lab using the CEBAF Large Acceptance Spectrometer (CLAS) [3]. CLAS provides the large angular coverage that is necessary for the study of resonance decays. It is a magnetic spectrometer based on a six-coil torus magnet whose field is primarily oriented along the azimuthal direction. The particle detection system includes drift chambers for track reconstruction, scintillation counters for the time of flight measurement, Cerenkov counters for electron-pion discrimination, and electromagnetic calorimeters to identify electrons and neutrals. Charged particles can be detected and identified for momenta down to $0.2 \mathrm{GeV}$.

CLAS is the first large acceptance instrument that can measure exclusive electroproduction of mesons with sufficient resolution for a detailed study of resonance excitation. The entire resonance mass region and a wide range in the photon virtuality $Q^{2}$ can be covered simultaneously in a single measurement while detecting several meson final states. Data taking with the CLAS detector started in 1998 and since that time many different reactions has been studied. In the following I will discuss some of the most recent and relevant results obtained from these analyses.

\section{Quadrupole deformation of the $\Delta(1232)$}

The $\Delta(1232)$ is the first excited state of the nucleon. It is a well identified and isolated resonance which dominates the photoproduction cross section and the electroproduction cross section at low momentum transfer $Q^{2}$. For this 
reason, this is probably the most studied of the nucleon excited states. In model with SU(6) spherical symmetry, the $N \rightarrow \Delta$ transition is simply due to a magnetic dipole $M_{1+}$ mediated by a spin flip from the $J=1 / 2$ nucleon ground state to the $\Delta$ with $J=3 / 2$, while the electric and coulomb multipoles $E_{1+}$ and $S_{1+}$, which are allowed by parity and angular momentum conservation, are equal to 0 . Non-zero values of $E_{1+}$ and $S_{1+}$ indicate a deviation from the SU(6) spherical symmetry that can be associated with a quadrupole deformation of the nucleon or of the $\Delta$ state as discussed in Ref. [4]. Dynamically such deformation may arise through interaction of the photon with the pion cloud $[5,6]$ or through the one-gluon-exchange mechanism [7]. At large momentum transfer, helicity conservation directly implies $R_{E M}=+1$. An interpretation of $R_{E M}$ in terms of quadrupole deformation can therefore only be valid at low momentum transfer.

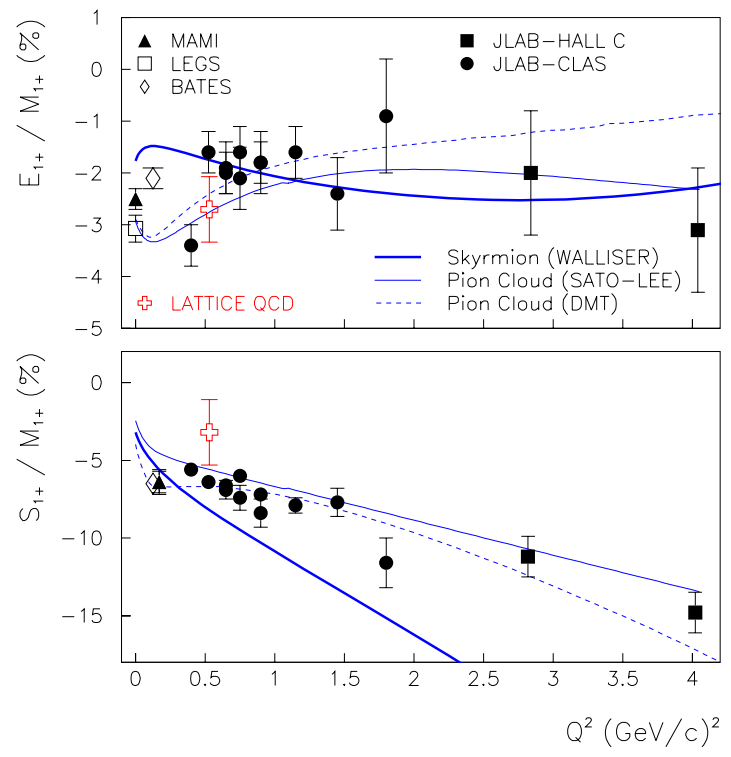

Figure 1. $R_{E M}$ and $R_{S M}$ as a function of $Q^{2}$. The CLAS data are shown in comparison with the results of previous experiments published after 1990. The results of a recent Lattice QCD calculation are also shown.

Results of the multipole analysis of the CLAS data [8] are shown in Fig. 1, where data from previous experiments published after 1990 are included as well [9-11]. $R_{E M}$ remains negative and small throughout the explored $Q^{2}$ range. There are no indications that leading $\mathrm{pQCD}$ contributions are important since they would result in a rise of $R_{E M}=E_{1+} / M_{1+} \rightarrow+1$ [12]. $R_{S M}$ behave quite differently. While it also remains negative, its magnitude tends to increase with $Q^{2}$. The comparison with theoretical models, from relativized quark models $[13,14]$ to a chiral quark soliton model [15] and dynamical models [5,6,16], shows that simultaneous description of both $R_{E M}$ and $R_{S M}$ is achieved by dynamical models that include explicitly the pion cloud. This supports the claim that most of the quadrupole strength is due to meson effects that are not included in other models. Recently, calculation of $R_{E M}$ and $R_{S M}$ have been performed in quenched and unquenched lattice QCD in the $Q^{2}$ range of the CLAS results [17]. The full QCD results give $R_{E M}$ values more negative than in the quenched approximation showing the contribution of the pion cloud to be negative, and causing an oblate deformation of the $\Delta(1232)$. The calculation at $Q^{2}=0.52 \mathrm{GeV}^{2}$ is in agreement with the CLAS data for $R_{E M}$ and $R_{S M}$.

\section{The second resonance region}

The mass region corresponding to the second enhancement in the inclusive $\gamma N$ cross section is known as the second resonance region. It covers the $W$ range between approximately $1.4 \mathrm{GeV}$ and $1.6 \mathrm{GeV}$ and is dominated by the excitation of the $S_{11}(1535)$ and $D_{13}(1520)$ states.

\subsection{The $S_{11}(1535)$ and $\eta$ production}

The $S_{11}(1535)$ resonance was found to have an unusually hard transition form factor. This in fact shows a slow falloff with $Q^{2}$. This state has a significant branching ratio in $p \eta$ final states where it shows up as a strong enhancement near the $\eta$ threshold with very little background. Older data show some discrepancies in the values of the total width and photocoupling amplitude. In particular, analysis of pion photoproduction data [1] disagree significantly with the analysis of $\eta$ photoproduction.

Data from CLAS [18] together with data from an earlier JLab experiment [19] now give a consistent picture of the $Q^{2}$ evolution of the form factor, confirming the slow fall-off with much improved data quality (see Fig. 2). Analysis of $n \pi^{+}$and $p \pi^{0}$ data at $Q^{2}=0.4 \mathrm{GeV}^{2}$ gives a value of $A_{1 / 2}$ consistent with the analysis of the $p \eta$ data [20].

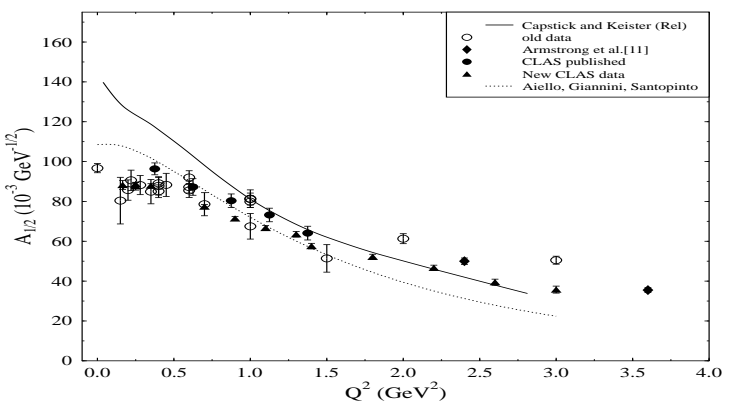

Figure 2. Transverse helicity amplitude $A_{1 / 2}\left(Q^{2}\right)$ for the $S_{11}(1535)$.

So far the particular hardness of the form factor has been difficult to explain in theoretical models. However a recent calculation in the framework of the constituent quark model has shown that this behavior can be reproduced using an hypercentral potential [21]. 


\subsection{Polarization Observables and Resonance amplitudes}

The extraction of the helicity amplitudes in an unpolarized measurement requires a complex analysis of the full angular distribution. On the contrary polarization measurements

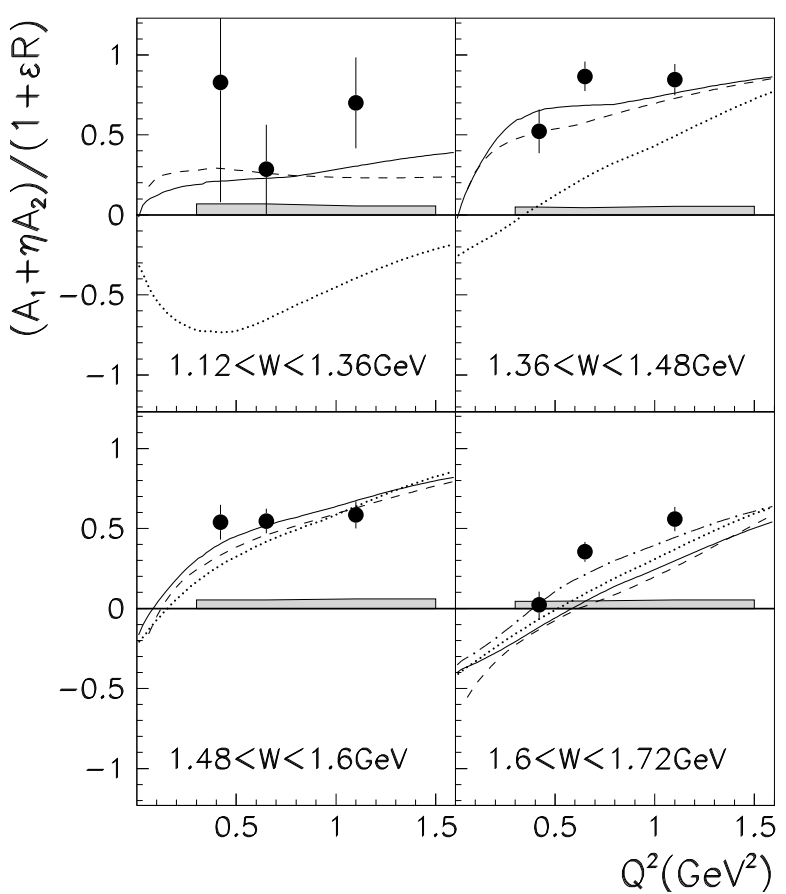

Figure 3. $Q^{2}$ dependence of the double spin asymmetry $\left(A_{1}+\right.$ $\left.\eta A_{2}\right) /(1+\epsilon R)$ for $\vec{e} \vec{p} \rightarrow e^{\prime} n \pi^{+}$. The data are compared with the pure resonance contribution (dotted line) predicted by the $\mathrm{AO}$ model [22], with the MAID [23] (dashed line) and AO (solid line) full calculations.

provide direct information on the helicity amplitudes $A_{1 / 2}$ and $A_{3 / 2}$ through the measurement of the double spin asymmetry.

The $Q^{2}$ dependence of the double spin asymmetry asymmetry $\left(A_{1}+\eta A_{2}\right) /(1+\epsilon R)$ for $\vec{e} \vec{p} \rightarrow e^{\prime} n \pi^{+}$is shown in Fig. 3 for four $W$ ranges [24]. $A_{1}$ is the virtual photon helicity asymmetry $A_{1}=\left(\left|A_{1 / 2}\right|^{2}-\left|A_{3 / 2}\right|^{2}\right) /\left(\left|A_{1 / 2}\right|^{2}+\right.$ $\left.\left|A_{3 / 2}\right|^{2}\right), A_{2}$ is a longitudinal-transverse interference term, $R$ is the longitudinal-transverse cross section ratio, while $\eta$ and $\epsilon$ are kinematical factors. Due to the particular kinematics of the experiments as well as to the suppression of longitudinal terms in respect to transverse, the double spin asymmetry is expected to be dominated by the helicity asymmetry $A_{1}$. The sign and magnitude of the measured asymmetry indicate the dominance of the helicity- $1 / 2$ contribution. This is in contrast with the helicity-3/2 dominance observed at the photon point [1] and indicates that a transition occurs in between $Q^{2}=0$ and the measured $Q^{2}$ range. This feature is consistent with a strong change with $Q^{2}$ of the helicity structure of the $D_{13}(1520)$ and $F_{15}(1680)$ states that are predicted by constituent quark models $[13,21,25,26]$ to vary from $A_{1}=-1$ at the photon point to $A_{1}=1$ at high $Q^{2}$.

\section{Higher mass states and missing res- onances}

The mass region above $W=1.5-1.6 \mathrm{GeV}$ is characterized by the presence of several excited states. Many of these states are expected to strongly decouple from the $\pi N$ final state. Therefore the measurement of other final states as $\pi \pi N, \eta N, \omega N, \ldots$ is very important. Moreover many of the so-called " missing states" are predicted to couple strongly to the $\pi \pi N$ channels [27]. Search for these states is of great importance for the understanding of the nucleon structure as alternative symmetry schemes do not predict nearly as many missing states [2].
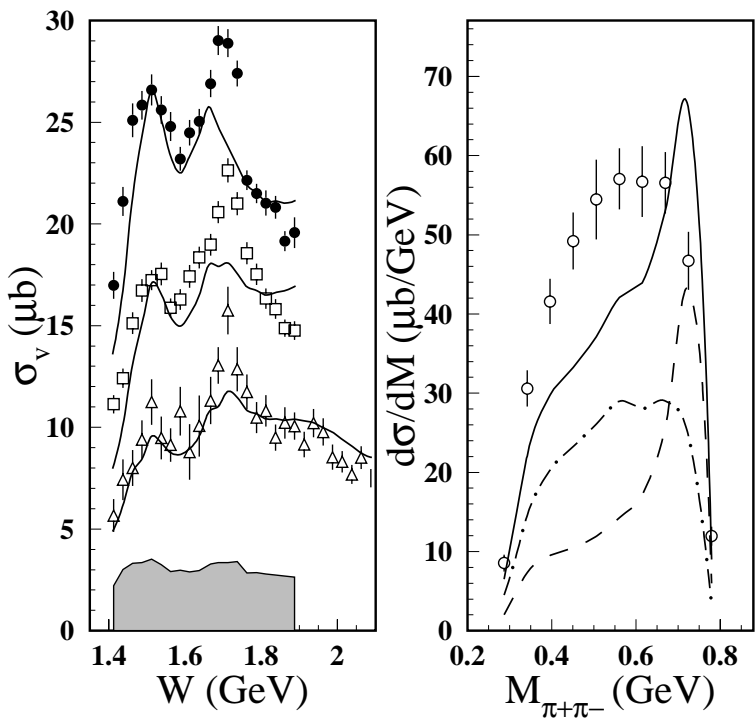

Figure 4. Left: Total cross section for $\gamma^{*} p \rightarrow p \pi^{+} \pi^{-}$as a function of $W$ at $<Q^{2}>\sim 0.65 \mathrm{GeV}^{2}$ (full points), $<Q^{2}>\sim$ $0.95 \mathrm{GeV}^{2}$ (open sqares) and $<Q^{2}>\sim 1.3 \mathrm{GeV}^{2}$ (open trangles). Right: $d \sigma / d M_{\pi^{+} \pi^{-}}$at $<Q^{2}>\sim 0.95 \mathrm{GeV}^{2}$. The curves represent the predictions of the phenomenological model of Ref. [28]. The dashed line includes resonances only, the dot-dashed the non-resonant part while the solid lines are the full calculation.

\subsection{Resonances in the $p \pi^{+} \pi^{-}$channel}

The cross section for the reaction $\gamma^{*} p \rightarrow p \pi^{+} \pi^{-}$has been measured at CLAS in $Q^{2}$ range from 0.5 to $1.5 \mathrm{GeV}^{2}$ [29]. The CLAS data are shown in Fig. 4. The most striking feature is the strong resonance peak near $W=1.72 \mathrm{GeV}$ that was not seen in the DESY photoproduction data [30-32]. Further analysis of the CLAS data that includes the complete hadronic angular distribution and the $p \pi^{+}$and $\pi^{+} \pi^{-}$ mass distributions has allowed us to investigate the origin of this peak. This was found to be better described by a $N_{3 / 2}^{*}(1720)$ state. While there exists a state with such quantum numbers in this mass range, the $P_{13}(1720)$, its hadronic properties as obtained from analysis of previous data seem to be inconsistent with what observed in this experiment. Keeping the hadronic coupling of this state within the limits imposed by previous data forces us to reduce its photocouplings and to introduce a second state with the same 
quantum numbers but very different hadronic couplings. Interpretation of this second state as a missing resonance is definitely possible. There are in fact model predictions of states with the same quantum numbers and reasonably close mass $[27,33]$.

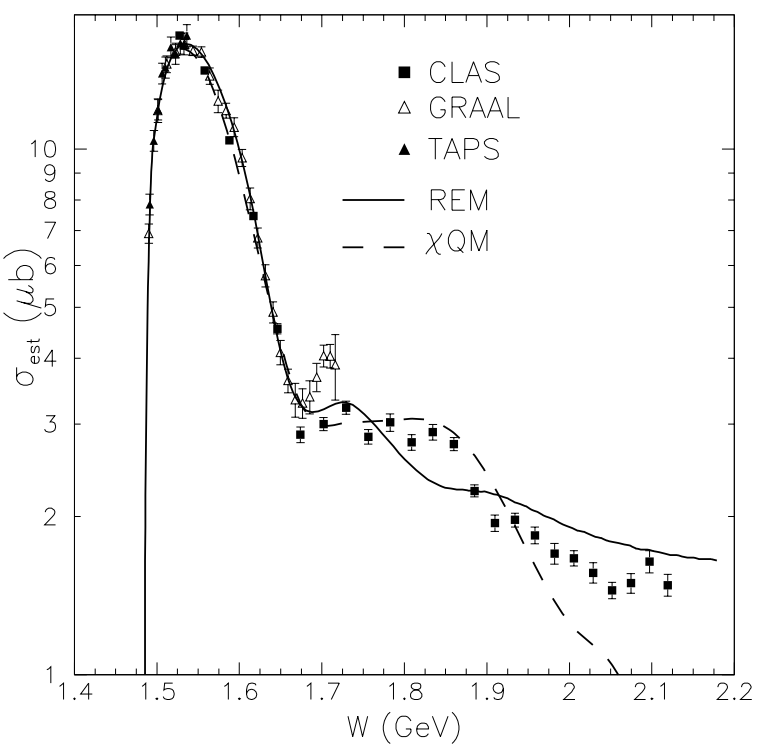

Figure 5. $R_{E M}$ and $R_{S M}$ as a function of $Q^{2}$. The CLAS data are shown in comparison with the results of previous experiments published after 1990. The results of recent Lattice QCD calculation are also shown.

\subsection{Photoproduction of $\eta$ meson}

The $\gamma p \rightarrow p \eta$ reaction has been measured with the CLAS detector covering the resonance region up to $W=2.15 \mathrm{GeV}$ [34]. The differential cross section as been measured almost in the entire angular range and the total cross section has been extracted. This is shown in Fig. 5. Beyond the region of the well known $S_{11}(1535)$, the data show a structure indicative of a higher mass resonance contribution to the $p \eta$ channel. Preliminary calculations based on the $\chi \mathrm{QM}$ [35] shows that good agreement for $W<1.9 \mathrm{GeV}$ is obtained when a new $S_{11}$ resonance with a mass of $1.79 \mathrm{GeV}$ and width of $250-350 \mathrm{MeV}$ is included. Further analysis of the angular distributions and the energy dependence are needed to come to a more definite conclusion.

\section{Conclusion}

Electroexcitation of baryon resonances has become an efficient tool to study the nucleon structure. The simultaneous measurement of several decay channels over a wide kinematics is necessary for a thorough study of resonance excitation. This is the goal of the measurements performed with the CLAS detector that provide new high quality data for many reactions covering the entire resonance region. These data have allowed us to significatly improve our understanding of the characteristics of known resonance states as the $\Delta(1232)$ or the $S_{11}(1535)$. Furthermore they now provide indications for the existence of new states which may allow us to solve the puzzle of the "missing" states.

\section{References}

[1] D. E. Groom et al., Eur. Phys. J C 15, 1 (2000).

[2] M. Kirchbach, Mod. Phys. Lett. A, 12, 3177 (1997).

[3] B. Mecking et al., Nucl. Instr. and Meth. , 94, 262 (2003).

[4] A. Buchmann and E. Henley, Phys. Rev. D , 65, 07317 (2002).

[5] T. Sato and T. S. Lee, Phys. Rev. C , 64, 055201 (2001).

[6] S. S. Kamalov and S. N. Yang, Phys. Rev. Lett., 83, 4494 (1999).

[7] R. Koniuk and N. Isgur, Phys. Rev. D, 21, 1868 (1980).

[8] K. Joo et al. (CLAS collaboration), Phys. Rev. Lett., 88, 122001 (2002).

[9] author R. Beck et al. , Phys. Rev. C, 61, 035204 (2000).

[10] G. Blanpied et al., Phys. Rev. C, 64, 025203 (2001).

[11] V. V. Frolov et al., Phys. Rev. Lett., 82, 45 (1999).

[12] G. A. Warren and C. E. Carlson, Phys. Rev. D, 42, 3020 (1990).

[13] M. Warns et al., Z. Phys. C, 45 , 627 (1990).

[14] I. G. Aznaurian, Z. Phys. A, 346, 297 (1993).

[15] A. Silva et al., Nucl. Phys. A, 675, 637 (2000).

[16] S. S. Kamalov et al. , Phys. Rev. C, 64, 033201 (2001).

[17] C. Alexandrou et al., hep-lat/0309041.

[18] R. Thompson et al. (CLAS Collaboration), Phys. Rev. Lett., 86, 1702 (2001).

[19] C. S. Armstrong et al., Phys. Rev. D , 60, 052004 (1999).

[20] I. G. Aznaurian et al., to be submitted to Phys. Rev. C.

[21] M. M. Giannini and E. Santopinto and A. Vassallo, Nucl. Phys. A, 699, 308 (2002).

[22] V. Burkert and Z. Li, Phys. Rev. D, 47, 46 (1993).

[23] D. Drechsel et al., Nucl. Phys. A 645, 145 (1999).

[24] R. De Vita et al. (CLAS Collaboration), Phys. Rev. Lett., 88, 082001 (2002).

[25] F. E. Close and Z. Li, Phys. Rev. D, 42, 2194 (1990).

[26] S. Capstick, Phys. Rev. D , 46, 2864 (1992).

[27] S. Capstick and W. Roberts, Phys. Rev. D, 49, 4570 (1994).

[28] V. Mokeev et al., Phys. of Atomic Nucl., 64, 1292 (2001).

[29] M. Ripani et al. (CLAS Collaboration), Phys. Rev. Lett., 91, 022002 (2003).

[30] V. Eckart et al., Nucl. Phys. B, 55, 45 (1973).

[31] P. Joos et al., Phys. Lett. B, 52, 481 (1974).

[32] K. Wacker et al., year 1978 , Nucl. Phys. B, 144, 269 (1978).

[33] S. Capstick and P. R. Page, Phys. Rev. D, 60, 111501 (1999).

[34] M. Dugger et al. (CLAS Collaboration), Phys. Rev. Lett., 89, 222002 (2002).

[35] B. Saghai, nucl-th/0202007 